\title{
Evaluation of Left Ventricular Assist Device Electrical Current as a Predictor of Left Ventricular Recovery
}

Dmitry V. Telyshev*1,2, Alexander A. Pugovkin ${ }^{1}$, Ivan A. Ephimov ${ }^{1}$, Aleksandr Markov ${ }^{2}$, Steffen Leonhardt ${ }^{3}$, Marian Walter ${ }^{3}$, Jamshid H. Karimov ${ }^{4}$, Sergey V. Selishchev ${ }^{1}$

${ }^{1}$ Institute of Biomedical Systems, National Research University of Electronic Technology, Zelenograd, 124498, Moscow, Russian Federation

${ }^{2}$ Institute for Bionic Technologies and Engineering, I. M. Sechenov First Moscow State Medical University, 119991, Moscow, Russian Federation

${ }^{3}$ Helmholtz Institute of Biomedical Engineering, RWTH Aachen University, 52074 Aachen, Germany

${ }^{4}$ Department of Biomedical Engineering, Lerner Research Institute, Cleveland Clinic, Cleveland, Ohio, OH 44195 USA 
Abstract: In this paper, we aim to assess the electric current parameters and report the analysis of the associated degree of ventricular recovery during left ventricular assist device (LVAD) support. An assumption was made there is a linear relationship between ventricular recovery degree and the pump electric current pulsatility index (PI). The experimental study was carried out using the ViVitro Pulse Duplicator System with Sputnik 1 LVAD connected. Cardiac output (CO) and cardiac power output (CPO) were used as a measure of ventricular recovery degree. Different heart rates (HR) $(59,73$, $86 \mathrm{bpm}$ ) and pump speeds (7600-8400 rpm in $200 \mathrm{rpm}$ steps) were investigated. Ventricular stroke volumes in the range of $30-80 \mathrm{ml}$ for each heart rate at certain pump speed were used. The obtained relationships of $\mathrm{CO}$ and $\mathrm{CPO}$ vs. PI was linear as the coefficients of determination for each regression curve were more than 0.8 . CO vs. PI: $\mathrm{R}^{2}=0.9218 ; 0.9271 ; 0.9172$ and $\mathrm{CPO}$ vs. PI: $\mathrm{R}^{2}=0.8517 ; 0.841 ; 0.8244$ for $\mathrm{HR}=59 \mathrm{bpm}$; $73 \mathrm{bpm} ; 86 \mathrm{bpm}$, respectively. Study findings suggest that adequate interpretation of parameters could potentially serve as a valuable clinical tool to assess ventricular recovery based on LVAD infrastructure without requiring any special hemodynamic assessment. Key words: heart failure, left ventricular assist device, cardiac recovery, mechanical circulation 


\section{Introduction}

Heart transplantation for heart failure patients who are not amenable to medical or surgical therapy remains the gold standard treatment for end-stage heart failure. However, the lack of organ donors will always limit the availability of heart transplants for the majority of the end-stage heart failure population. As an alternative, the use of mechanical circulatory support (MCS) devices has increased in recent decades and has become a valid clinical option. [1]

Although the overall usage of continuous-flow (CF) left ventricular assist devices (LVADs) continues to increase, therapy management aspects remain challenging, with no consensus on welldefined assessment criteria, support duration, and particularly, explantation strategies. [2-7] In past decades it was numerously reported that CF LVAD support can lead to cardiac recovery. [3$5,8,9]$ As the technology advances, instrumentation for ventricular recovery evaluation becomes crucial in improving of patient outcomes. Proper recovery evaluation leads to better overall assessment and helps to develop treatment and explantation strategies in patients supported with MCS.

Today's ventricular assist devices (VADs) mainly use continuous-flow pump technology. [10,11] The only moving part in such pumps is an impeller driven by a brushless DC motor. The main parameters that need to be constantly assessed under continuous-flow operating conditions to implement physiological pump control are the blood flow through the pump, the pressure at the pump inlet (preload) and the pressure at the pump outlet (afterload). $[12,13]$ Output parameters like blood flow rate and pressure drop in the pump result from the controlled impeller rotation speed. Two methods to determine the blood flow rate can be used: 1) a direct measurement, and 2) an estimation through indirect methods using intrinsic LVAD parameters to derive flow rate via a process model. $[7,12,14-20]$ However, the second approach has limitations in accuracy caused by the low system efficiency of rotary blood pumps, which makes it difficult to assess the direct correlation between the electric and hydraulic parameters of the system. [7,19-22] For instance, 
the maximum efficiency of continuous-flow rotary pump typically does not exceed $30 \%$. [23-26] Such a low efficiency can be explained by various energy losses in the whole system, i.e., by electric, mechanical and hydraulic energy losses.

The relationship between the pump power consumption and blood flow rate has been extensively studied and reported by our group. [27] This relationship was studied for two generations of continuous-flow rotary pumps: 1) the first generation of Sputnik LVAD that has been successfully implemented into clinical practice, [28] and 2) the second generation development, which was aimed at miniaturization and reducing the risk of blood cell trauma. [21] The nonlinear relation between blood flow rate and power consumption demonstrates that the minimum power consumption cannot be obtained at the point of zero flow in the pump. [14] The described relationship shows that estimation of the blood flow in axial pumps using indirect methods does not reliably determine the blood flow rate in lower range values.

The recovery evaluation protocol is based primarily on echocardiographic assessment, and adequate evaluation is often impeded by quality of visualization, user subjectivity, mechanical ventilation, swelling in the early postoperative period and variability of hemodynamic conditions in patients during assessment of ventricular function while on LVAD support. [29]

In this paper, we aim to assess the electric current parameters and report the analysis of the associated degree of left ventricular recovery during LVAD support. An assumption was made that there is a linear relationship between ventricular recovery degree and the pulsatility index of the pump electric current.

The interpretation of the LVAD parameters related to electric current could have a valuable clinical predictive value by allowing ventricular recovery assessment with possibility to avoid additional hemodynamic measurements. The proposed predictive tool could also be useful for monitoring the effectiveness of LVAD support and report the analysis of the associated degree of left ventricular recovery during LVAD support. 


\section{Materials and Methods}

Experiment setup and device description. The experimental study was carried out using the ViVitro Pulse Duplicator System (PDS, SD2001-1; ViVitro Inc., Victoria, BC, Canada) that allows simulation of various hemodynamic conditions for adult and pediatric patients. (Figure 1, with a Sputnik 1 LVAD, an axial-flow blood pump with a nonpulsatile flow that achieves a flow of up to 10 1/min., connected). The PDS consists of a hydraulic circuit based on the ViVitro Heart Model, the SuperPump pulsatile pump, an ultrasonic flow sensor (ME-11PXL Clamp-on Tubing Flow sensors; Transonic Systems, Ithaca, NY, USA) and the ViViTest data acquisition system.
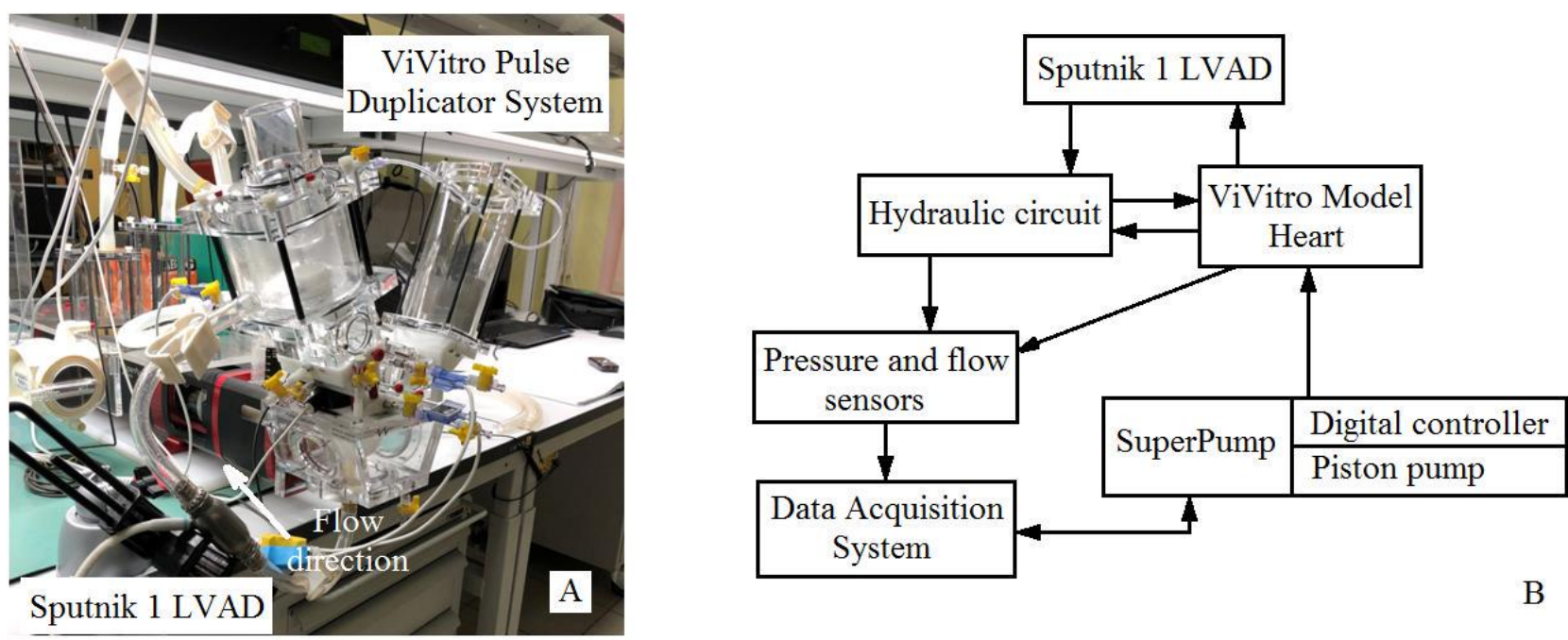

Figure 1. Image (A) of ViVitro Pulse Duplicator System SD2001-1 with the Sputnik 1 LVAD connected $(B)$ and the block diagram

The Sputnik 1 LVAD features technical performance parameters that are on the same level as or better than a HeartMate II or a HeartAssist 5 VAD Systems. [30] The dimensions of the pump are: length, $81 \mathrm{~mm}$; maximum diameter, $34 \mathrm{~mm}$; impeller diameter, $15.6 \mathrm{~mm}$; and pump weight, 246 g.

The SuperPump pulsatile pump is a digitally controlled hydraulic piston pump generating physiological cardiac flows. Digital control allows the operator to set the stroke volume (SV) of 
the ViVitro Model Heart, which generates the physiological cardiac output (CO) in the hydraulic circuit at a pre-set heart rate. The ViVitro Model Heart is an acrylic chamber divided into two parts (piston pump and hydraulic circuit) by the silicon membrane and is driven by the pulsatile pump (SuperPump) in order to reproduce left ventricle (LV) contraction.

The hydraulic circuit of the circulatory mock loop consists of:

- An aortic unit including an aortic root and an aorta which are adjustable compliance chambers;

- A systemic vascular unit reproducing the physiological resistance of the systemic vasculature, and;

- An atrium unit, which represents an open chamber, with a fluid level corresponding to an atrium pressure level.

The ultrasonic flow sensor is connected to a signal converter to validate the flow measurements. The signal from the system, along with signals from three pressure sensors connected to the ViVitro Model Heart aortic and atria, are transmitted to the ViViTest data acquisition system. In combination with the ViViTest software, the system allows the operator to record and analyze the data of physiological flows and pressures in the hydraulic circuit, and also to adjust the control signal of the SuperPump pulsatile pump.

A glycerol-water mixture with a dynamic viscosity of $3.5 \mathrm{mPa} \cdot \mathrm{s}$ at $22.0^{\circ} \mathrm{C}$ was used in the hydraulic circuit to reproduce the blood viscosity of a patient with an implanted Sputnik 1 LVAD. In this study, we investigated different heart rates (HR), i.e., 59, 73 and $86 \mathrm{bpm}$, and pump speeds ranging from 7600 to $8400 \mathrm{rpm}$ in $200 \mathrm{rpm}$-increment steps. This particular speed range was chosen because it allows the operator to maintain the blood flow through the pump in the range of 1 to 7 liters per minute. Ventricular stroke volumes in the range of $30-80 \mathrm{ml}$ for each heart rate value at a certain pump speed were used. It is consistent to gradually changing cardiac states from 
end-stage heart failure to normal state including recovery. Characteristics of each state were simulated according to clinical data reported in the recent work of Jakovljevic, et al. [8]

Systolic, diastolic and mean aortic pressures were obtained using the ViViTest software. Additionally, the mean aortic pressure was calculated using the following equation:

$$
P_{\text {mean }}=P_{d}+0,412 \times\left(P_{s}-P_{d}\right)
$$

where $P_{d}$ and $P_{s}$ are the diastolic aortic pressure and the systolic aortic pressure, respectively.

Cardiac output and cardiac power output (CPO) were used as a measure of ventricular recovery degree. CPO is the hydraulic energy required by the heart to provide enough blood flow to the systemic circulation. [9] $\mathrm{CO}$ and $\mathrm{CPO}$ were calculated as follows [8]:

$$
\begin{gathered}
C O=H R \times S V, \\
C P O=0,00222 \times P_{\text {mean }} \times C O .
\end{gathered}
$$

During experiments with the Sputnik 1 LVAD, the electric current values were calculated based on power consumption $(W)$ and supply voltage $(U)$ acquired via the electronic control unit of the LVAD:

$$
I=\frac{W}{U}
$$

The pulsatility index (PI) of the Sputnik 1 LVAD electric current was calculated as follows:

$$
P I=\frac{I_{\max }-I_{\min }}{I_{\text {mean }}}
$$

where $I_{\max }, I_{\min }$ and $I_{\text {mean }}$ are maximum, minimum and mean values of the electric current averaged over all cardiac cycles during one simulated hemodynamic condition. 
Parameters of the pump. A block diagram of the LVAD biotechnical system is shown in Figure

2. It represents the following parameters:

- $\quad P_{1}$ and $P_{2}$ are pressures in the left ventricle and aorta, respectively.

- $\quad Q_{1}, Q_{2}$, and $Q_{3}$ are the blood flow rates through the aortic valve, continuous-flow rotary pump, and aorta, respectively.

- $\quad H$ represents the head pressure across the pump.

- $\quad I$ is the electric current measured in the motor windings.

- $A$ is the external work of the left ventricle.

- $\omega$ is the rotation speed of the pump set to continuous flow mode.

Among the described parameters, it is necessary to highlight the following equations:

$$
\begin{gathered}
Q_{3}=Q_{1}+Q_{2} \\
H=P_{2}-P_{1},
\end{gathered}
$$

Control issues of the LVAD presented in Figure 2 include: 1) the discrepancy between the parameters that need to be controlled ensuring circulatory support (i.e., $P_{1}, P_{2}, Q_{1}, Q_{3}$ ); 2 ) the parameters that continuous-flow rotary pump generates $\left(H, Q_{2}\right)$; and, 3) the parameters that can be tracked and changed in real time during LVAD support $(I, \omega)$. It was previously shown that there are ways to find correlations between different parameters using: 1) a ratio between maximum flow acceleration and flow pulsatility at baseline pump speed as an alternative to a regression coefficient between maximum flow acceleration and flow pulsatility at different pump speeds [29]; 2) the external work of the ventricle expressed by the area inside the P-V diagrams correlates with the area inside dynamic H-Q curves [31]; or 3) energetic characteristics of LV-LVAD interaction. [32] In our work, it is hypothesized that changes in the work $(A)$ of the left ventricle affect the 
recorded parameter of the electric current $(I)$. In particular, it is assumed that by changing $I$, it is possible to evaluate the myocardial function recovery.

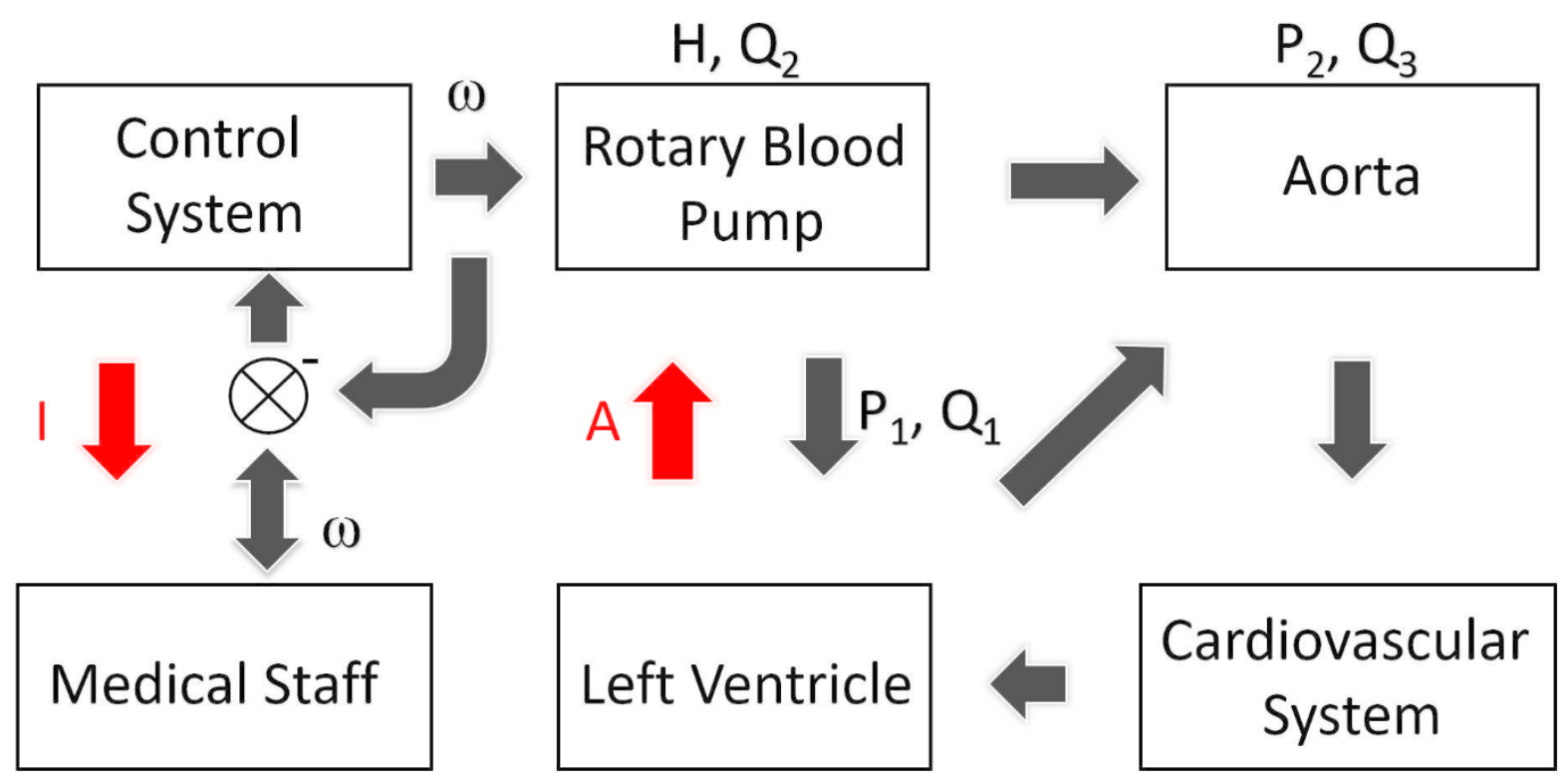

Figure 2. Biotechnical block diagram of LVAD. H, head pressure across the pump; $Q_{1}$, aortic valve flow rate; $Q_{2}$, pump flow rate; $Q_{3}$, aortic flow rate to systemic circulation; $P_{1}$, pump inlet pressure; $P_{2}$, pump outlet pressure; $\omega$, rotation speed of the pump impeller; I, pump electric current measured in the motor windings; A, external work of the left ventricle. Red arrows indicate parameters under the study, black arrows indicate other parameters of circulation supported with LVAD.

In order to investigate the effect of cardiac muscle recovery on the pump electric current pulsatility index (PI), a comparison of cardiac output and cardiac power output characteristics vs. PI was performed. The obtained data was processed using a regression analysis.

\section{Results}

Figure 3 represents the data of the cardiac output and cardiac power output, depending on the pulsatility index of the Sputnik 1 LVAD electrical current, for three different heart rate values.

Typical relationships between CO and CPO vs. PI for different heart rate values $(59,73$ and 86 bpm) are show in Figure 3A and 3B, respectively. These relationships tend to be linear, especially in the case of $\mathrm{CO}$ values (Figure 3A). Coefficients of determination for each heart rate value are 
given in the plot to highlight the linearity. Even though these coefficients are smaller for CPO (Figure 3B) in comparison to $\mathrm{CO}$, the linearity is still visible and obvious. This allows us to infer that an increase in PI indicates a linear increase in cardiac output. Therefore, as the pump operational mode was not changed, it can be assumed that a cardiac recovery takes place. Moreover, Figure 3 shows that slope of the regression curve increases with increasing heart rate.

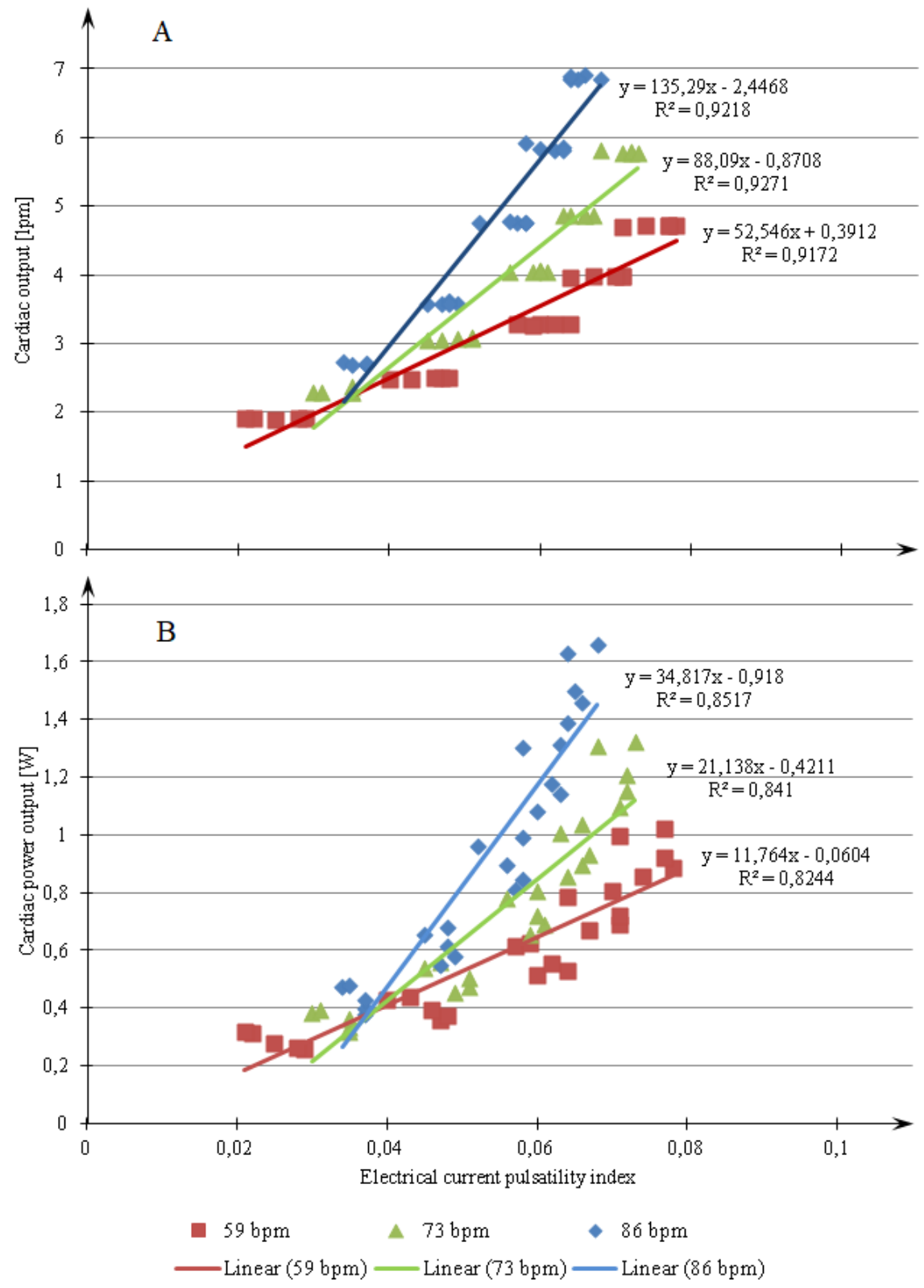

Figure 3. Cardiac output (A) and cardiac power output (B) as a function of Sputnik 1 LVAD electrical current pulsatility index for three heart rate values: 59 (red), 73 (green) and 86 (blue) bpm. Linear regression equations and coefficients of determination are given by the curves. 
Represented criterion variables are the recovery measure of the left ventricle with LVAD implanted, which have linear correlation with the Sputnik 1 LVAD electric current pulsatility index. Thus, recovery degree can be evaluated by this index only with possibility to avoid other hemodynamic measurement.

\section{Discussion}

In this work, we used the Sputnik 1 LVAD as a research object in experiments. Assuming that the use of LVADs may result in myocardial recovery, it is imperative to be able to accurately evaluate parameters of the LV chamber during hemodynamic measurements. Moreover, finding reliable correlations between different devices and hemodynamic parameters will be necessary, despite potential complications attributable to various inconsistencies that may vary from mechanical aspect to electrical (windings losses, rotor losses, friction loss, etc.). However, precise estimation may be a limiting factor in this case. Nevertheless, provided that these estimations were performed in a correct and meticulous manner, it is possible to evaluate the correlation between the pulsatility index of electric current and cardiac muscle recovery.

According to numerous reports, patients in end-stage heart failure are able to recover cardiac function with a combination of LVAD and medical therapies. [3-6,8,9,29] It was noted that the cardiac function of patients with an explanted LVAD can reach levels equivalent to those of healthy controls. The recovery degree was evaluated during hemodynamic measurements at rest and in response to physical exercise testing in LVAD-implanted patients. [8] A sufficient degree of recovery may serve as an indication of effectiveness of LVAD therapy, or as an indicator to start explantation procedures.

The main parameters that need to be controlled while providing circulatory support are the pressure drop in the pump and blood flow rate. In order to control the blood flow rate and the pressure drop 
in modern LVADs, indirect estimation methods are used, based on the analysis of such parameters as the electric current, power consumption, [15] blood viscosity, [16] rotation speed of LVAD impeller, torque, hematocrit level and Reynolds number. [17,18]

It is possible to reliably and precisely estimate the blood flow in centrifugal-type LVADs, since the power consumption depends linearly on the blood flow rate and the pressure drop. In axialtype LVADs, this relationship is characterized by a non-linear, non-monotonic function, which allows reliable estimation of the blood flow rate only over 2 liters per minute. $[14,18]$

In this study, the major goal was to determine the possibility of assessing the energy parameters of the heart depending on the recorded pump electric parameters. It should be noted that the purpose of the study was not to obtain an accurate regression model. The assumption was made that investigated relationships between $\mathrm{CO}$ or $\mathrm{CPO}$ and PI can be linear, and the results indicate the validity of the assumption. However, from the graphs presented, it can be seen that the slope of the relationship changes with increasing value of the pulsatility index of the pump electric current, which indicates the non-linear nature of the relationship. This concern will be taken into account in the further work.

\section{Limitations}

This study has a number of limitations which should be noted. The possibility of mitral regurgitation (MR) was not considered, despite the clinically observed MR improvement following LVAD implantation in end-stage heart failure patients. [7] Also the study lacks of specificity/sensitivity analysis and evaluation of possible sources of error. A linear relationship between heart recovery and pump current is due to the other factors that can alternate pump current.

Only the mechanical activity of the left ventricle specific for sinus rhythm was studied. The electrophysiological measurements were not collected. Validation of the results in a broad range of hemodynamic conditions will be addressed once we move closer towards in vivo studies. 
Since the presented evaluation method requires a sufficient increase in accuracy and the study lacks of clinical data, its relevance for the predictability of clinical performance is still to be proven.

\section{Conclusion}

Investigation of electric current parameters and report on the associated degree analysis of left ventricular recovery during LVAD support were performed in this study. The linear relationship between cardiac output or cardiac power output and the pulsatility index of electric current was also explored. Study findings suggest that adequate interpretation of parameters could potentially serve as a valuable clinical tool to assess ventricular recovery and assist with strategies of device explantation possibly without requiring any special hemodynamic assessment. The unique feature of the proposed methodology is that the system feedback should enable continuous monitoring of ventricular function, based on LVAD infrastructure.

The general methodology presented in this paper may be applied to any continuous flow LVAD, provided the specific characteristics to compute the used indices are known. Further work needs to address this generalization claim and test the method for different designs. 


\section{Acknowledgments}

The reported study was funded by Russian Foundation for Basic Research (RFBR) research project № 19-51-12005 and Deutsche Forschungsgemeinschaft (DFG, Project 409796053).

\section{Author Contributions:}

D.T. conceived the research idea. D.T. and S.S. supervised and coordinated the research. D.T. and S.S. designed the pump. I.E. and A.P. collected the data and conducted the statistical expertise. D.T., and A.M. are responsible for the literature search. A.M., and D.T. wrote the manuscript with input from all authors. D.T., A.M., M.W., S.L. and J.K. performed the critical review of the article.

\section{Conflict of interest}

The authors declare that they have no conflict of interest. 


\section{References}

1. Kirklin, J.K.; Pagani, F.D.; Kormos, R.L.; Stevenson, L.W.; Blume, E.D.; Myers, S.L.; Miller, M.A.; Baldwin, J.T.; Young, J.B.; Naftel, D.C. Eighth annual INTERMACS report: Special focus on framing the impact of adverse events. J. Hear. Lung Transplant. 2017, 36, 1080-1086.

2. Kado, Y.; Miyamoto, T.; Fukamachi, K.; Karimov, J.H. Update on the management and associated challenges of adult patients treated with veno-arterial extracorporeal membrane oxygenation. Expert Rev. Med. Devices 2019, 16, 483-491.

3. Hetzer, R.; Müller, J.; Weng, Y.; Wallukat, G.; Spiegelsberger, S.; Loebe, M. Cardiac recovery in dilated cardiomyopathy by unloading with a left ventricular assist device. Ann. Thorac. Surg. 1999, 68, 742-749.

4. Frazier, O.H.; Baldwin, A.C.W.; Demirozu, Z.T.; Segura, A.M.; Hernandez, R.; Taegtmeyer, H.; Mallidi, H.; Cohn, W.E. Ventricular reconditioning and pump explantation in patients supported by continuous-flow left ventricular assist devices. J. Hear. Lung Transplant. 2015, 34, 766-772.

5. Burkhoff, D.; Klotz, S.; Mancini, D.M. LVAD-Induced Reverse Remodeling: Basic and Clinical Implications for Myocardial Recovery. J. Card. Fail. 2006, 12, 227-239.

6. Moscato, F.; Schima, H. Mechanical Circulatory Support in End-Stage Heart Failure; Montalto, A., Loforte, A., Musumeci, F., Krabatsch, T., Slaughter, M.S., Eds.; Springer International Publishing: Cham, 2017; ISBN 978-3-319-43381-3.

7. Swetz, K.M.; Freeman, M.R.; Mueller, P.S.; Park, S.J. Clinical management of continuousflow left ventricular assist devices in advanced heart failure. J. Hear. Lung Transplant. 2010, 29, 1081.

8. Jakovljevic, D.G.; Yacoub, M.H.; Schueler, S.; MacGowan, G.A.; Velicki, L.; Seferovic, P.M.; Hothi, S.; Tzeng, B.-H.; Brodie, D.A.; Birks, E.; et al. Left Ventricular Assist Device as a Bridge to Recovery for Patients With Advanced Heart Failure. J. Am. Coll. Cardiol. 2017, 69, 1924-1933.

9. Hall, S.G.; Garcia, J.; Larson, D.F.; Smith, R. Cardiac power index: Staging heart failure for mechanical circulatory support. Perfus. (United Kingdom) 2012, 27, 456-461.

10. Moazami, N.; Fukamachi, K.; Kobayashi, M.; Smedira, N.G.; Hoercher, K.J.; Massiello, A.; Lee, S.; Horvath, D.J.; Starling, R.C. Axial and centrifugal continuous-flow rotary pumps: A translation from pump mechanics to clinical practice. J. Hear. Lung Transplant. 2013, 32, 1-11.

11. Ferrari, M.; Kruzliak, P.; Spiliopoulos, K. An insight into short- and long-term mechanical circulatory support systems. Clin. Res. Cardiol. 2014, 104, 95-111.

12. Giridharan, G.A.; Skliar, M. Physiological Control of Blood Pumps Using Intrinsic Pump Parameters: A Computer Simulation Study. Artif. Organs 2006, 30, 301-307.

13. Giridharan, G.A.; Skliar, M. Control Strategy for Maintaining Physiological Perfusion with Rotary Blood Pumps. Artif. Organs 2003, 27, 639-648.

14. Pennings, K.A.M.A.; Martina, J.R.; Rodermans, B.F.M.; Lahpor, J.R.; Van De Vosse, F.N.; De Mol, B.A.J.M.; Rutten, M.C.M. Pump flow estimation from pressure head and power uptake for the heartassist5, heartmate II, and heartware vads. ASAIO J. 2013, 59, 420-426.

15. Ayre, P.J.; Lovell, N.H.; Woodard, J.C. Non-invasive flow estimation in an implantable rotary blood pump: a study considering non-pulsatile and pulsatile flows. Physiol. Meas. 2003, 24, 179-189.

16. Malagutti, N.; Karantonis, D.M.; Cloherty, S.L.; Ayre, P.J.; Mason, D.G.; Salamonsen, R.F.; Lovell, N.H. Noninvasive Average Flow Estimation for an Implantable Rotary Blood Pump: A New Algorithm Incorporating the Role of Blood Viscosity. Artif. Organs 2007, 31, 45-52.

17. Wakisaka, Y.; Okuzono, Y.; Taenaka, Y.; Chikanari, K.; Masuzawa, T.; Takano, H. 
Establishment of Flow Estimation for an Implantable Centrifugal Blood Pump. ASAIO J. 1997, 43, M663.

18. Boës, S.; Thamsen, B.; Haas, M.; Daners, M.S.; Meboldt, M.; Granegger, M. Hydraulic Characterization of Implantable Rotary Blood Pumps. IEEE Trans. Biomed. Eng. 2019, 66, 1618-1927.

19. Pauls, J.P.; Stevens, M.C.; Bartnikowski, N.; Fraser, J.F.; Gregory, S.D.; Tansley, G. Evaluation of Physiological Control Systems for Rotary Left Ventricular Assist Devices: An In-Vitro Study. Ann. Biomed. Eng. 2016, 44, 2377-2387.

20. Wu, Y. Adaptive Physiological Speed/Flow Control of Rotary Blood Pumps in Permanent Implantation Using Intrinsic Pump Parameters. ASAIO J. 2009, 55, 335-339.

21. Telyshev, D.; Denisov, M.; Pugovkin, A.; Selishchev, S.; Nesterenko, I. The Progress in the Novel Pediatric Rotary Blood Pump Sputnik Development. Artif. Organs 2018, 42, 432443.

22. Telyshev, D.; Denisov, M.; Markov, A.; Fresiello, L.; Verbelen, T.; Selishchev, S. Energetics of blood flow in Fontan circulation under VAD support. Artif. Organs 2020, 44, 50-57.

23. Nguyen, D.Q.; Thourani, V.H. Third-Generation Continuous Flow Left Ventricular Assist Devices. Innov. Technol. Tech. Cardiothorac. Vasc. Surg. 2010, 5, 250-258.

24. Loree, H.M.; Bourque, K.; Gernes, D.B.; Scott Richardson, J.; Poirier, V.L.; Barletta, N.; Fleischli, A.; Foiera, G.; Gempp, T.M.; Schoeb, R.; et al. The HeartMate III: Design and in vivo studies of a Maglev centrifugal left ventricular assist device. Artif. Organs 2001, 25, 386-391.

25. Pagani, F.D. Continuous-Flow Rotary Left Ventricular Assist Devices with "3rd Generation” Design. Semin. Thorac. Cardiovasc. Surg. 2008, 20, 255-263.

26. Tansley, G.; Vidakovic, S.; Reizes, J. Fluid dynamic characteristics of the VentrAssist rotary blood pump. Artif. Organs 2000, 24, 483-487.

27. Selishchev, S. V.; Telyshev, D. V. Optimisation of the sputnik-VAD design. Int. J. Artif. Organs 2016, 39, 407-414.

28. Selishchev, S. V.; Telyshev, D. V. Ventricular assist device sputnik: Description, technical features and characteristics. Trends Biomater. Artif. Organs 2015, 29, 207-210.

29. Gupta, S.; Muthiah, K.; Woldendorp, K.; Robson, D.; Jansz, P.; Hayward, C.S. Derivation of indices of left ventricular contractility in the setting of continuous-flow left ventricular assist device support. Artif. Organs 2014, 38, 1029-1034.

30. Chiu, W.-C.; Girdhar, G.; Xenos, M.; Alemu, Y.; Soares, J.S.; Einav, S.; Slepian, M.; Bluestein, D. Thromboresistance Comparison of the HeartMate II Ventricular Assist Device With the Device Thrombogenicity Emulation-Optimized HeartAssist 5 VAD. J. Biomech. Eng. 2014, 136, 021014.

31. Yokoyama, Y.; Kawaguchi, O.; Kitao, T.; Kimura, T.; Steinseifer, U.; Takatani, S. Prediction of the external work of the native heart from the dynamic H-Q curves of the rotary blood pumps during left heart bypass. Artif. Organs 2010, 34, 766-777.

32. Pugovkin, A.A.; Markov, A.G.; Selishchev, S. V; Korn, L.; Walter, M.; Leonhardt, S.; Bockeria, L.A.; Bockeria, O.L.; Telyshev, D. V Advances in Hemodynamic Analysis in Cardiovascular Diseases Investigation of Energetic Characteristics of Adult and Pediatric Sputnik Left Ventricular Assist Devices during Mock Circulation Support. Cardiol. Res. Pract. 2019, 2019, 1-15. 